\title{
极小正则 2-图及其应用*
}

范红兵

( Department of Physics and Computer Science, Wilfrid Laurier University, Waterloo, N2L 3C5, Canada)

\section{刘桂真**}

(山东大学数学与系统科学学院, 济南 250100)

\section{刘季平}

( Department of Mathematics and Computer Science, University of Lethbridge, Lethbridge, T1K 3M4, Canada)

摘要 2- 图是边的尺寸至多为 2 的超图, 极小正则2-图是不含有真正则因子 的正则 2- 图. 设 $f_{2}(n)$ 为所有 $n$ 个顶点的极小正则 2- 图的最大度数. 给出了极 小正则 2- 图的一个结构性质, 并由此证得 $f_{2}(n)=(n+3-i) / 3$, 其中 $1 \leqslant i \leqslant 6$, $n \geqslant 7, i \equiv n(\bmod 6)$, 从而解决了范红兵等人提出的一个猜想. 作为在图论中的 应用, 可以刻画不可分解因子的正则图, 并给出关于度条件的最好可能的因子 存在性定理. 进而, $f_{2}(n)$ 和极小2-图可应用于最初引发这项研究的通用开关盒 设计问题.

\section{关键词 图 正则因子 2-图 开关盒设计}

\section{1 引言}

考虑边的尺寸至多为 2 的多重超图，并称它们为 2- 图，因此，无环图是没 有单边 (尺寸为 1 的边) 的 2- 图. 这种关系有助于将 2- 图转换成图, 因而可以应 用图的理论.

2005-08-02 收稿

*加拿大国家自然科学基金、中国国家自然科学基金 (批准号: 10471078) 和高等学校博士学科专项 基金 (批准号: 20040422004) 资助项目

**联系人, E-mail: gzliu@sdu.edu.cn 
若一个 2- 图的每个顶点恰好包含在 $k$ 条边里, 则称该 2- 图是 $k$-正则的. 2图 $G$ 的一个 $k$ - 因子 (或 $k$-正则因子) 是 $G$ 的一个 $k$-正则支撑子图. 若一个正则 2- 图不含有真正则因子, 则称它为极小的 (或不可分解的, 或不可分解因子的). 不含 2- 因子的 2-图 $G$ 称为是无 2- 因子的; 若它的边可分解成 2- 因子, 则它是 2- 因子可分解的. 2- 图 $G$ 的一个分支是指它的一个极大 2-边连通子图. 2- 图的 分支可以包含被单边覆盖的某些顶点, 但是, 当去掉那些单边时, 该分支就变成 2- 边连通的 (和图中一样). 设 $S$ 是一个集合, $k$ 是非负整数, 用 $S^{k}$ 记 $S$ 中每个元 素都出现 $k$ 次的多重集合. 若 $S=\emptyset$ 或 $k=0$, 则 $S^{k}=\emptyset$. 其他有关图和超图的术 语和符号可分别参见文献 [1] 和 [2].

在一般的通用开关模型 ${ }^{[3]}$ 的研究中, 我们必须枚举出 $n$ 个顶点的所有正则 2-图. 这可以如下进行: 设

$$
\mathcal{P}_{2}=\{S|S \subset\{1,2, \cdots, n\}, 1 \leqslant| S \mid \leqslant 2\},
$$

则 $\left|\mathcal{P}_{2}\right|=n(n+1) / 2$. 设 $A=\left(a_{i j}\right)$ 是 $\mathcal{P}_{2}$ 的一个邻接矩阵, 使得当 $i \in S_{j}$ 时, $a_{i j}=1$, 否则 $a_{i j}=0$, 其中 $S_{j}$ 是 $\mathcal{P}_{2}$ (按某个顺序) 的第 $j$ 个集合. 设 $B$ 是矩 阵 $A$ 的增广矩阵, 使得最后一列元素全为 -1 , 则 $B$ 是一个 $n \times m$ 矩阵, 其中 $m=(n(n+1)+2) / 2$. 设 $X=\left(x_{1}, \cdots, x_{m}\right)^{\mathrm{T}}$. 线性 Diophantos 方程系统 (LDES)

$$
B X=0
$$

的任意非负整数解均给出一个 $x_{m}$ 正则 2 - 图, 其顶点集合为 $\{1,2, \cdots, n\}$, 边集合 为 $\left\{S_{1}^{x_{1}}, S_{2}^{x_{2}}, \cdots, S_{m-1}^{x_{m}-1}\right\}$. 反之亦然.

设 $\left(x_{1}, \cdots, x_{m}\right)$ 和 $\left(y_{1}, \cdots, y_{m}\right)$ 是方程 (1) 的两个非负整数解, 并设 $X$ 和 $Y$ 分别是与它们对应的 2- 图. 当且仅当对所有的 $i=1, \cdots, m$ 均有 $x_{i} \leqslant y_{i}$ 时, 定义 $\left(x_{1}, \cdots, x_{m}\right) \preceq\left(y_{1}, \cdots, y_{m}\right)$. 于是当且仅当 $X$ 是 $Y$ 的一个正则因子时, $\left(x_{1}, \cdots, x_{m}\right) \preceq\left(y_{1}, \cdots, y_{m}\right)$. 由 Higman 的定理 ${ }^{[4]}$, 加上序 $\preceq$ 的非负整数解集是一 个好的半序集，因此，它有一个有限基. 容易看到: LDES (1) 的极小非负整数解 集是一个基, 它对应于 $n$ 个顶点的极小正则 2- 图集, 记作 $\mathcal{G}_{2}(n)$, 因此，我们必 须描述 $\mathcal{G}_{2}(n)$ 中元素的特征, 并且要将每个 $n$ 个顶点的正则 2- 图表示成 $\mathcal{G}_{2}(n)$ 中 不交图的并. WQO 理论保证了 $\mathcal{G}_{2}(n)$ 的有限性. 设

$$
f_{2}(n)=\max \left\{r \mid \exists G \in \mathcal{G}_{2}(n), G \text { 的度为 } r\right\},
$$

则 $f_{2}(n)$ 是确切定义的.

一个 LDES 的极小非负整数解集也叫做 Hilbert 基, 已知它是可计算的 ${ }^{[5]}$, 因 此, 可以通过计算方程 (1) 的 Hilbert 基来枚举 $\mathcal{G}_{2}(n)$ 的元素. 已经有几个算法用 以计算 LDES 的 Hilbert 基. Contejean 和 Devie ${ }^{[5]}$ 综述了先前的几个算法, 并给出 一个有效的渐近算法. 虽然所有这些算法都可用以计算 $\mathcal{G}_{2}(n)$ 和 $f_{2}(n)$, 但它们不 能提供 $f_{2}(n)$ 的明确表达式和计算 $\mathcal{G}_{2}(n)$ 的令人满意的效率.

文献 [3] 中提出了以下猜想: 
猜想 $1.1 f_{2}(n)=(n+3-i) / 3$, 其中 $n \geqslant 7,1 \leqslant i \leqslant 6, i \equiv n(\bmod 6)$.

本文中, 我们直接研究 2- 图. 应用图论中的理论和方法, 首先确立了极小 正则 2- 图的结构特征, 据此确定了函数 $f_{2}(n)$, 从而证明了该猜想. 通过应用正则 图的特性, 我们获得关于度条件的最好可能的因子存在性定理. 最后给出 $f_{2}(n)$ 和极小 2- 图在通用开关盒设计中的一个应用.

\section{2 极小 2- 图的特征}

本节中, 首先给出正则图的一个结构性质, 然后用它刻画极小 2- 图的特征.

设 $G=(V, E)$ 是一个图, $D, S \subset V$ 是不交的子集. 用 $G-D$ 记从 $G$ 中删除 $D$ 中的所有顶点所得到的图, $d_{G-D}(x)$ 表示图 $G-D$ 中顶点 $x$ 的度. 称 $G-D$ 的有奇数个顶点的分支为奇分支. 用 $E_{G}(S, D)$ 记 $G$ 中一个端点在 $S$ 中, 另一端 点在 $D$ 中的边的集合, $e_{G}(S, D)$ 记 $E_{G}(S, D)$ 中边的数目, 即

$$
e_{G}(S, D)=\left|E_{G}(S, D)\right| .
$$

在我们的证明中将用到下面已知的结果:

引理 2.1 ${ }^{[6]}$ 图 $G$ 有 1- 因子当且仅当对任意的 $S \subset V(G)$,

$$
o(G-S) \leqslant|S|,
$$

其中 $o(G-S)$ 表示 $G-S$ 的奇分支的数目.

引理 2.2 ${ }^{[7]}$ 每个没有割边的 3- 正则图都有 1- 因子.

引理 2.3 ${ }^{[7]}$ 任一 $2 k$ - 正则图都是 2- 因子可分解的.

引理 2.4 ${ }^{[8]}$ 一个多重图 $G$ 含有一个 $k$ - 因子当且仅当

$$
\Delta=k|D|-q(D, S)-\sum_{x \in S}\left(k-d_{G-D}(x)\right) \geqslant 0
$$

对所有不相交集合 $D, S \subset V(G)$ 均成立，其中 $q(D, S)$ 表示满足 $e_{G}(S, V(C))+$ $k|V(C)|$ 是奇数的 $G-D-S$ 的那些分支 $C$ 的数目.

引理 2.5 ${ }^{[8]}$ 设 $G$ 是一个没有割边的 3- 正则图, $e_{1}, e_{2}$ 是 $G$ 的任意两条边, 则 $G^{\prime}=G-\left\{e_{1}, e_{2}\right\}$ 含有 1 - 因子.

下面的定理将用来证明我们的主要定理:

定理 2.1 设 $G$ 是一个 $(2 r+1)$ - 正则图 $(r \geqslant 1)$. 若 $G$ 中割边的数目少于 $2 r+1$, 则 $G$ 有 $2-$ 因子.

证 首先证明 $r=1$ 的情形. 假定 $G$ 是一个有 $k$ 条割边的 3- 正则图 $(k \leqslant 2)$. 若 $k=0$, 由引理 2.2, $G$ 有 1- 因子, 因而 $G$ 含有 2- 因子.

若 $k=1$, 再次证明 $G$ 有 1 - 因子, 从而证得它有 2- 因子. 假定结论不成立, 则由引理 2.1, 存在一个 $S \subset V(G)$, 使得 $o(G-S)>|S|$. 注意到: 对 $G-S$ 的每个 奇分支 $C$ 而言, $e_{G}(C, S)$ 必定是奇数; $G$ 仅有一条割边, 因而至多有一个奇分支 $C$ 具有 $e_{G}(C, S)=1$, 其他的奇分支 $C$ 具有 $e_{G}(C, S) \geqslant 3$, 于是

$$
3(o(G-S)-1)+1 \leqslant 3|S|
$$


或者

$$
3 o(G-S)-2 \leqslant 3|S| .
$$

这蕴含着 $o(G-S) \leqslant|S|$, 矛盾.

现在设 $k=2$. 用反证法证明 $G$ 含有 2- 因子. 当从 $G$ 中删除那两条割边时, 必定有一个分支, 设为 $H$, 不含有 2 - 因子. 进而, 注意到那两条割边必定都与 $H$ 邻接. 若不然, 用一条割边连接两个 $H$ 可以得到一个图 $H^{\prime}$. 由上一段的证明, $H^{\prime}$ 有 2- 因子, 这意味着 $H$ 有 2- 因子, 而这与 $H$ 的选择矛盾. 设 $u_{1}$ 和 $u_{2}$ 是与 $G$ 中那两条割边相邻接的 $H$ 中的两个顶点, 则 $u_{1}, u_{2}$ 是相异的, 在 $H$ 中的度都 为 2 . 设 $K$ 是删除顶点 $u_{1}$ 和 $u_{2}$ 并添加边 $e_{1}^{\prime}$ 和 $e_{2}^{\prime}$ 分别连接 $u_{1}$ 和 $u_{2}$ 各自邻点所 得到的 3-正则图, 则 $H$ 含有 2- 因子当且仅当 $K$ 有包含边 $e_{1}^{\prime}$ 和 $e_{2}^{\prime}$ 的 2- 因子, 这 当且仅当 $K$ 有不覆盖 $e_{1}^{\prime}$ 和 $e_{2}^{\prime}$ 的 1- 因子. 由引理 2.5 知 $K$ 有不覆盖 $e_{1}^{\prime}$ 和 $e_{2}^{\prime}$ 的 1因子, 因此 $H$ 有 2- 因子, 这又与 $H$ 的选择矛盾. 当 $r=1$ 时, 定理证毕.

下面设 $r \geqslant 2$. 估计 (2) 式中所定义的 $\Delta$ 的值. 对任意两个不相交的集合 $D, S \subset V(G)$, 我们有

$$
\begin{aligned}
\Delta & =2|D|-q(D, S)-\sum_{x \in S}\left(2-d_{G-D}(x)\right) \\
& =2|D|-q(D, S)-2|S|+\sum_{x \in S} d_{G-D}(x) \\
& =2|D|-q(D, S)-2|S|+(2 r+1)|S|-e_{G}(D, S) \\
& =2|D|-2|S|+(2 r+1)|S|-e_{G}(D, S)-q(D, S) .
\end{aligned}
$$

对 $e_{G}(V(C), S)+2|V(C)|$ 为奇数的 $G-D-S$ 的一个分支 $C$ 而言, $e_{G}(V(C), S)$ 是奇数. 设 $\mathcal{A}$ 是 $G-D-S$ 的分支 $C$ 的集合, 满足 $e_{G}(V(C), S)$ 是奇数并且 $e_{G}(V(C), D)=0$. 于是, 对 $\mathcal{A}$ 中的每一分支 $C$, 若没有一条割边连接 $C$ 和 $S$, 则 $S$ 和 $C$ 之间至少有三条边. 设 $\mathcal{B}$ 是 $G-D-S$ 的分支 $C$ 的集合, 满足 $e_{G}(V(C), S)$ 是奇数并且 $e_{G}(V(C), D) \geqslant 1$, 则 $\mathcal{A} \cap \mathcal{B}=\emptyset$, 并且 $q(D, S)=|\mathcal{A}|+|\mathcal{B}|$. 有 3 种可能 的情形:

情形 $1|D| \geqslant|S|$.

$G$ 是 $(2 r+1)$ - 正则的, 所以

$$
q(D, S)+e_{G}(D, S) \leqslant(2 r+1)|S|,
$$

因而

$$
\Delta=2(|D|-|S|)+\left[(2 r+1)|S|-e_{G}(D, S)-q(D, S)\right] \geqslant 0 .
$$

情形 $2|D|<|S|$ 并且 $2|S|-2|D| \leqslant|\mathcal{A}|-(2 r-1)$.

首先注意到 $|\mathcal{A}| \geqslant 2 r+1$. 设 $\mathcal{A}_{1}$ 是仅有一条割边连接 $S$ 的分支的集合. 因为 $G$ 没有多于 $2 r$ 条割边, 于是

$$
\left|\mathcal{A}_{1}\right| \leqslant 2 r, \quad \mathcal{A}_{2}=\mathcal{A}-\mathcal{A}_{1} \neq \emptyset .
$$

这样, 


$$
\begin{aligned}
\Delta & =2|D|-2|S|+(2 r+1)|S|-e_{G}(D, S)-(|\mathcal{A}|+|\mathcal{B}|) \\
& =(2 r+1)|S|-e_{G}(D, S)-(|\mathcal{A}|+|\mathcal{B}|+2|S|-2|D|) \\
& \geqslant(2 r+1)|S|-e_{G}(D, S)-[|\mathcal{A}|+|\mathcal{B}|+|\mathcal{A}|-(2 r-1)] \\
& =(2 r+1)|S|+(2 r-1)-e_{G}(D, S)-(2|\mathcal{A}|+|\mathcal{B}|) \\
& =(2 r+1)|S|+2 r-1-2\left(\left|\mathcal{A}_{1}\right|+\left|\mathcal{A}_{2}\right|\right)-e_{G}(D, S)-|\mathcal{B}| \\
& \geqslant(2 r+1)|S|-e_{G}(D, S)-|\mathcal{B}|-\left(\left|\mathcal{A}_{1}\right|+3\left|\mathcal{A}_{2}\right|\right)+2 r-1-\left|\mathcal{A}_{1}\right|+\left|\mathcal{A}_{2}\right| \\
& =2 r-1+\left|\mathcal{A}_{2}\right|-\left|\mathcal{A}_{1}\right| \\
& \geqslant 0 .
\end{aligned}
$$

情形 $3|D|<|S|$ 并且 $2|S|-2|D|>|\mathcal{A}|-(2 r-1)$.

若 $|S|-|D| \geqslant 2$, 则

$$
\begin{aligned}
\Delta & =2|D|-2|S|+(2 r+1)|S|-e_{G}(D, S)-(|\mathcal{A}|+|\mathcal{B}|) \\
& =2|D|-2|S|+(2 r+1)|S|-e_{G}(D, S)-[|\mathcal{A}|-(2 r-1)]-|\mathcal{B}|-(2 r-1) \\
& \geqslant 2|D|-2|S|+(2 r+1)|S|-e_{G}(D, S)-[2|S|-2|D|-1]-|\mathcal{B}|-(2 r-1) \\
& =(2 r-3)|S|-\left[\left(e_{G}(D, S)+|\mathcal{B}|\right)-4|D|\right]-(2 r-2) \\
& \geqslant(2 r-3)|S|-[(2 r+1)|D|-4|D|]-(2 r-3)-1 \\
& =(2 r-3)(|S|-|D|-1)-1 \\
& \geqslant 0 .
\end{aligned}
$$

若 $|\mathcal{A}|$ 是奇数, 则

$$
|\mathcal{A}|-(2 r-1) \leqslant 2|S|-2|D|-2,
$$

并有

$$
\begin{aligned}
\Delta & =2|D|-2|S|+(2 r+1)|S|-e_{G}(D, S)-(|\mathcal{A}|+|\mathcal{B}|) \\
& =2|D|-2|S|+(2 r+1)|S|-e_{G}(D, S)-[|\mathcal{A}|-(2 r-1)]-|\mathcal{B}|-(2 r-1) \\
& \geqslant 2|D|-2|S|+(2 r+1)|S|-e_{G}(D, S)-(2|S|-2|D|-2)-|\mathcal{B}|-(2 r-1) \\
& =(2 r-3)|S|-\left[\left(e_{G}(D, S)+|\mathcal{B}|\right)-4|D|\right]-(2 r-3) \\
& \geqslant(2 r-3)|S|-[(2 r+1)|D|-4|D|]-(2 r-3) \\
& =(2 r-3)(|S|-|D|-1) \\
& \geqslant 0 .
\end{aligned}
$$

如果

$$
e_{G}(D, S)+|\mathcal{B}| \leqslant(2 r+1)|D|-1
$$

那么

$$
\Delta=2|D|-2|S|+(2 r+1)|S|-e_{G}(D, S)-(|\mathcal{A}|+|\mathcal{B}|)
$$




$$
\begin{aligned}
& =2|D|-2|S|+(2 r+1)|S|-e_{G}(D, S)-[|\mathcal{A}|-(2 r-1)]-|\mathcal{B}|-(2 r-1) \\
& \geqslant 2|D|-2|S|+(2 r+1)|S|-e_{G}(D, S)-[2|S|-2|D|-1]-|\mathcal{B}|-(2 r-1) \\
& =(2 r-3)|S|-\left[\left(e_{G}(D, S)+|\mathcal{B}|\right)-4|D|\right]-(2 r-2) \\
& \geqslant(2 r-3)|S|-[(2 r+1)|D|-1-4|D|]-(2 r-2) \\
& \geqslant(2 r-3)(|S|-|D|-1) \\
& =0 .
\end{aligned}
$$

现在我们可以假设

$$
|S|=|D|+1, \quad e_{G}(D, S)+|\mathcal{B}|=(2 r+1)|D|
$$

并且 $|\mathcal{A}|$ 是偶数.

如果 $|\mathcal{A}| \leqslant 2 r-1$, 那么有

$$
\begin{aligned}
\Delta & =2|D|-2|D|-2+(2 r+1)|D|+2 r+1-e_{G}(D, S)-(|\mathcal{A}|+|\mathcal{B}|) \\
& =(2 r+1)|D|+2 r-1-e_{G}(D, S)-(|\mathcal{A}|+|\mathcal{B}|) \\
& =(2 r+1)|D|+2 r-1-e_{G}(D, S)-(|\mathcal{A}|+|\mathcal{B}|) \\
& =2 r-1-|\mathcal{A}| \\
& \geqslant 0
\end{aligned}
$$

现在假定 $|\mathcal{A}|>2 r-1$, 我们证明这种情形不存在. 我们有

$$
\begin{gathered}
e_{G}(D, S)+|\mathcal{B}|=(2 r+1)|D|, \\
e_{G}(D, S)+|\mathcal{B}|+|\mathcal{A}| \leqslant(2 r+1)|S|=(2 r+1)|D|+2 r+1, \quad|\mathcal{A}| \leqslant 2 r+1 .
\end{gathered}
$$

$|\mathcal{A}|$ 是偶数, 因而有 $|\mathcal{A}|=2 r$. 于是

$$
e_{G}(D, S)+|\mathcal{B}|+|\mathcal{A}|=(2 r+1)|D|+2 r=(2 r+1)|S|-1 .
$$

存在唯一的一条边连接 $S$ 和 $\mathcal{A}$ 中的一个分支, 并且这条边必定是割边. 因为假 若存在分支 $C^{\prime} \in \mathcal{A}$, 使得 $e_{G}\left(C^{\prime}, S\right)>1$, 则由 $e_{G}\left(C^{\prime}, S\right)$ 是奇数有 $e_{G}\left(C^{\prime}, S\right) \geqslant 3$, 并且

$$
\begin{aligned}
(2 r+1)|S| & \geqslant\left|e_{G}\left(D \cup_{C \in \mathcal{A} \cup \mathcal{B}} C, S\right)\right| \\
& \geqslant e_{G}(D, S)+|\mathcal{B}|+|\mathcal{A}|+2 \\
& \geqslant(2 r+1)|D|+2 r+2 \\
& \geqslant(2 r+1)|S|+1,
\end{aligned}
$$

矛盾. 因此, $S$ 和 $\mathcal{A}$ 中的分支由 $2 r$ 条割边相连. $\mathcal{B}$ 中的每个分支和 $S$ 有奇数条 边相连, 因而这个数必定是 1 . 否则, 将会有

$$
\begin{aligned}
(2 r+1)|S| & \geqslant e_{G}\left(D \cup_{C \in \mathcal{A} \cup \mathcal{B}} C, S\right) \\
& \geqslant e_{G}(D, S)+|\mathcal{B}|+2+|\mathcal{A}|
\end{aligned}
$$




$$
\begin{aligned}
& \geqslant(2 r+1)|D|+2+2 r \\
& \geqslant(2 r+1)|S|+1,
\end{aligned}
$$

这又是一个矛盾, 因此,

$$
\left|e_{G}\left(D \cup_{C \in \mathcal{A} \cup \mathcal{B}} C, S\right)\right|=e_{G}(D, S)+|\mathcal{B}|+|\mathcal{A}|=(2 r+1)|S|-1 .
$$

于是有一条边 $e=u v \notin E_{G}\left(D \cup_{C \in \mathcal{A} \cup \mathcal{B}} C, S\right)$ 与 $S$ 邻接, 不妨设 $u \in S$. 如果 $v$ 不 属于 $S$, 那么 $u v$ 是 $G$ 的一条割边, 并且现在 $G$ 至少有 $2 r+1$ 条割边, 矛盾, 因 而 $v \in S$. 所以

$$
\begin{aligned}
(2 r+1)|S| & =\sum_{v \in S} d_{G}(v) \\
& \geqslant\left|E_{G}\left(D \cup_{C \in \mathcal{A} \cup \mathcal{B}} C, S\right)\right|+2 \\
& =e_{G}(D, S)+|\mathcal{B}|+|\mathcal{A}|+2 \\
& \geqslant(2 r+1)|S|+1 \\
& >(2 r+1)|S|,
\end{aligned}
$$

矛盾.

在任一情形, 我们都证得 $\Delta \geqslant 0$, 因此由引理 2.4, G 有 2- 因子. 定理证毕.

现在讨论 2- 图. 如果一个 2- 图 $G$ 有 1 个以上顶点被单边所覆盖, 则可以相 继去掉两条相异的单边, 并添加上被那两条单边所覆盖的两个顶点所形成的一 条新边, 以此得到仅有 1 个顶点被单边所覆盖的一个图或 2- 图. 这个新图记作 $\bar{G}$. 注意, 2- 图 $\bar{G}$ 不是唯一的, 但如果 $G$ 是极小的, 那么 $\bar{G}$ 也是极小的, 因此, 关 于 $f_{2}(n)$ 时, 我们只需要考虑至多 1 个顶点被单边覆盖的那些 2- 图, 在文献 [3] 中称其为本原 2- 图. 下面的引理是显然的:

引理 2.6 设 $G$ 是一个 2- 图. 若 $\bar{G}$ 有 2- 因子, 则 $G$ 也有; 若 $\bar{G}$ 是 2- 因子 可分解的, 则 $G$ 也是.

众所周知的 Peterson 的 2- 因子可分解定理 (引理 2.3) 可推广到 2- 图.

定理 2.2 任一 $2 k$ - 正则 2- 图是 2- 因子可分解的.

证 设 $G$ 是一个 $2 k$-正则 2 - 图, $k \geqslant 1$. 考虑图 $\bar{G}$. 若 $\bar{G}$ 不含有单边, 则由引 理 2.3, $\bar{G}$ 是 2- 因子可分解的, 因此由引理 2.6 得 $G$ 是 2- 因子可分解的.

现假定 $\bar{G}$ 有一条单边 $\{x\}$, 其重数 $p(x)>0$. 因为 $p(x)+2 e=2 k n$ 是偶数, $p(x)$ 必定是偶数, 其中 $e$ 是 $G$ 中尺寸为 2 的边的数目. 如果 $p(x)=2 k$, 那么 $\bar{G}-x$ 是一个 $2 k$-正则图, 由引理 2.3, 它是 2- 因子可分解的. 由此可得 $G$ 是 2- 因子可 分解的. 如果 $0<p(x)<2 k$, 我们从 $\bar{G}$ 中删除 $p(x)$ 条单边 $\{x\}$, 而后添加 3 个新 顶点 $x_{1}, x_{2}, x_{3}$ 和新边 $\left\{x, x_{1}\right\},\left\{x_{1}, x_{2}\right\},\left\{x_{1}, x_{3}\right\}$ 与 $\left\{x_{2}, x_{3}\right\}$, 它们的重数分别为 $p(x),(2 k-p(x)) / 2,(2 k-p(x)) / 2$ 及 $(2 k+p(x)) / 2$. 我们得到了一个 $2 k$ - 正则图 $G^{\prime}$. 又由引理 2.3, $G^{\prime}$ 是 2- 因子可分解的. 从 $G^{\prime}$ 的构造可以看出 $G^{\prime}$ 的一个 2- 因 子导出 $\bar{G}$ 的一个 2- 因子, 因此, $\bar{G}$ 是 2- 因子可分解的, $G$ 也是如此.

现在我们证明下面的定理, 它给出了极小 2- 图的特征: 
定理 2.3 设 $G$ 是一个极小 $k$-正则 2- 图, 则下列说法之一成立:

(i) $G$ 是 $1-$ 正则的;

(ii) $G$ 是 2- 正则的并含有一个奇圈;

(iii) $k(\geqslant 3)$ 是奇数且存在一个无 2 - 因子的分支 $C$, 使得若 $C$ 有奇数条重数 为 1 的单边并且没有重数大于 2 的单边, 则 $C$ 至少与 $G$ 的 $k-1$ 条割边邻接; 否 则, $C$ 至少与 $k$ 条割边邻接.

进而, 若 $G$ 是一个本原 $k$-正则 2- 图, 则上述条件 (i) (iii) 对 $G$ 是极小的也 是充分的.

证 设 $G$ 是一个 $k$-正则 2- 图. 当 $k=1$ 时, 容易看到 $G$ 是极小的当且仅当 $G$ 是一个 1 - 正则 2- 图. 当 $k=2$ 时, $G$ 由一些不相交的圈构成, 有些可能是重 数为 2 的单边, 有些可能是端点被重数为 1 的单边所覆盖的 $K_{2}$. 如果 $G$ 的连通 分支不是奇圈, 那么每个那样的分支都能分解成两个 1 - 因子, 因此 $G$ 是极小的 当且仅当它含有奇圈.

现在假定 $G$ 是一个极小 $k$-正则 2- 图, $k \geqslant 3$. 由定理 2.2, 可以假设 $k=$ $2 r+1, r \geqslant 1$. 考虑从 $G$ 中删除所有割边所得到的那个图, 它必定含有无 2- 因子 的分支 $C$. 因为若不然, $G$ 会含有 $2-$ 因子. 我们证明若 $C$ 有奇数条重数为一的 单边并且没有重数大于 2 的单边, 则 $C$ 至少与 $G$ 的 $2 r$ 条割边邻接, 否则, $C$ 至少与 $2 r+1$ 条割边邻接. 若不然, 设 $e_{1}, \cdots, e_{m}$ 是与 $C$ 邻接的所有割边, 并 设 $v_{1}, \cdots, v_{m}$ 是 $C$ 中分别与 $e_{1}, \cdots, e_{m}$ 邻接的那些顶点. 注意 $v_{1}, \cdots, v_{m}$ 可以不 是相异的. 首先我们假定 $C$ 有奇数条重数为 1 的单边, 并且没有重数大于 2 的 单边, 则任一 $\bar{C}$ 都有一个顶点, 设为 $x$, 被重数为 1 的一条单边所覆盖. 现在按 如下方式从 $\bar{C}$ 构造图 $C^{\prime}$ : 置 $x=v_{0}$. 对每个 $v_{i}(i=0,1, \cdots, m)$, 添加 3 个新顶点 $x_{i}, y_{i}, z_{i}$ 和边 $v_{i} x_{i},\left\{x_{i} y_{i}\right\}^{(r)},\left\{x_{i} z_{i}\right\}^{(r)},\left\{y_{i} z_{i}\right\}^{(r+1)}$, 则 $C^{\prime}$ 是一个 $(2 r+1)-$ 正则 图.

可以看出 $C^{\prime}$ 不含有 2- 因子, 否则 $\bar{C}$ 会含 2- 因子, 这又进一步说明 $C$ 不是 无 2- 因子的, 因此 $C^{\prime}$ 是具有 $(m+1)$ 条割边的无 2- 因子的 $(2 r+1)$ - 正则图. 由 定理 2.1 , 有 $m+1 \geqslant 2 r+1$ 或 $m \geqslant 2 r$. 这证明了 $C$ 至少与 $G$ 的 $2 r=k-1$ 条割边 邻接.

现在可以假设 $C$ 或者有偶数条重数为 1 的单边, 或者有重数至少为 3 的单 边 $\{x\}$. 在这种情形, 通过相继适当的连接两条单边, 总存在一个 $C^{\prime}$, 使得或者 $C^{\prime}$ 是一个图, 或者它含有重数至少为 2 的单边, 或者 $C^{\prime}$ 含有重数分别为 2 和 3 的两条单边. 现在按如下方式从 $C^{\prime}$ 构造 $C^{\prime \prime}$ :

首先, 对每个 $v_{i}(i=1, \cdots, m)$, 添加 3 个新顶点 $x_{i}, y_{i}, z_{i}$ 和边 $v_{i} x_{i},\left\{x_{i} y_{i}\right\}^{(r)}$, $\left\{x_{i} z_{i}\right\}^{(r)},\left\{y_{i} z_{i}\right\}^{(r+1)}$.

其次, 对每个具有奇重数 $p(v)$ 的单边 $\{v\}$ (注意 $3 \leqslant p(v)<k$ ), 删除 $p(v)$ 重 的 $\{v\}$, 而后添加 3 个新顶点 $y, z, w$ 到 $C^{\prime}$ 中, 并添加边 $v y, y z, y w$ 和 $z w$, 它们 
的重数分别为 $p(v),(k-p(v)) / 2,(k-p(v)) / 2$ 和 $(k+p(v)) / 2$.

第三，对每个具有偶重数 $p(u)$ 的单边 $\{u\}$ (注意 $p(u) \geqslant 2$ ), 删除 $p(u)$ 重的 $\{u\}$, 而后添加两个新顶点 $y^{\prime}, z^{\prime}$ 和新边 $v y^{\prime}, v z^{\prime}, y z^{\prime}$, 它们的重数分别为 $p(u) / 2$, $p(u) / 2$ 和 $k-\frac{p(u)}{2}$.

于是 $C^{\prime \prime}$ 是一个具有 $m$ 条割边的无 2- 因子的 $(2 r+1)$ - 正则图, 因此, 由定 理 2.1, $m \geqslant 2 r+1$, 从而 $C$ 至少与 $2 r+1=k$ 条割边邻接.

要完成定理 2.3 的证明, 还需要证明当 $G$ 是一个本原 $(2 r+1)$ - 正则 2- 图时, 定理 2.3 的条件 (iii) 对 $G$ 是极小的是充分的. 假定条件 (iii) 对无 2- 因子的分支 $C$ 成立, 并假定 $G$ 有一个真正则因子. 因为 $k$ 是奇数, $G$ 必定有一个偶正则因 子, 因而由定理 2.2, $G$ 有一个 2- 因子 $F$. 注意 $G$ 是本原的, 因而 $F$ 不含有任何 割边; 若 $F$ 含有某条单边 $\{v\}$, 它的重数至少为 2. 这个结果意味着限制在 $V(C)$ 上的 $F$ 是 $C$ 的一个 2 - 因子, 矛盾, 因此 $G$ 是极小的.

\section{3 函数 $f_{2}(k)$}

在这一节中，我们应用定理 2.3 来确定函数 $f_{2}(n)$, 从而证明文献 [3] 中的猜 想.

\section{定理 3.1}

$$
f_{2}(n)= \begin{cases}1, & 1 \leqslant n \leqslant 2, \\ 2, & 3 \leqslant n \leqslant 6, \\ (n+3-i) / 3, & n \geqslant 7,\end{cases}
$$

其中 $1 \leqslant i \leqslant 6$ 并且 $i \equiv n(\bmod 6)$.

证 若 $n=1$, 容易看出 $\mathcal{G}_{2}(1)$ 包括唯一的 2- 图, 其边集为 $\{\{1\}\}$, 因此 $f_{2}(1)=1$.

对 $n=2, \mathcal{G}_{2}(2)$ 包含两个正则 2- 图, 一个的边集合是 $\{\{1\},\{2\}\}$, 另一个的 边集合是 $\{\{1,2\}\}$, 因此 $f_{2}(2)=1$.

当 $3 \leqslant n \leqslant 6$ 时, 一个 3 - 圈加上一个 $(n-3)$ 个顶点的 2- 正则 2 - 图给出 $n$ 个 顶点的极小 2 - 正则 2- 图, 因此可以看出 $f_{2}(n) \geqslant 2$.

设 $n_{0}$ 是满足 $f_{2}\left(n_{0}\right) \geqslant 3$ 的最小整数, 并设 $G_{0}$ 是 $n_{0}$ 个顶点的极小 $3-$ 正则 $2-$ 图. 由引理 2.6 , 可以假设 $G_{0}$ 是本原的.

若 $G_{0}$ 不含单边, 则 $G_{0}$ 是图. 由定理 2.3, 得出 $G_{0}$ 有一个无 2- 因子的分支 $C$ 与至少 3 条割边邻接. 因为 $G_{0}$ 是无环的, 由一条割边与 $C$ 相连的每个分支都 至少有 3 个顶点, 因此

$$
n_{0} \geqslant|C|+3 \times 3 \geqslant 10 .
$$

假定 $G_{0}$ 含有单边 $\{x\}$. 如果 $\{x\}$ 的重数是 3 , 那么从 $G_{0}$ 中删除 $x$, 得到 $\left(n_{0}-1\right)$ 个顶点的 3-正则极小图. 由以上的论述, 我们有 $n_{0}-1 \geqslant 10$ 或者 $n_{0} \geqslant 11$. 如果 $\{x\}$ 的重数是 2 , 那么去掉两重 $\{x\}$, 另外添加两个顶点 $y, z$ 和边 $x y, x z,\{z y\}^{(2)}$, 
得到一个极小 3 - 正则图. 于是有 $n_{0}+2 \geqslant 10$ 或者 $n_{0} \geqslant 8$. 最后如果 $\{x\}$ 的重数 是 1 , 那么去掉 $\{x\}$, 另外添加 3 个顶点 $y, z, w$ 和边 $x y, y z, y w,\{z w\}^{(2)}$, 得到一 个极小 3 - 正则图. 于是我们有 $n_{0}+3 \geqslant 10$ 或者 $n_{0} \geqslant 7$.

总的来说, 我们有 $n_{0} \geqslant 7$. 这意味着当 $3 \leqslant n \leqslant 6$ 时, $f_{2}(n) \leqslant 2$, 因此当 $3 \leqslant n \leqslant 6$ 时, $f_{2}(n)=2$.

接下来证明 $f_{2}(n) \geqslant(n+3-i) / 3$, 其中 $1 \leqslant i \leqslant 6, i \equiv n(\bmod 6)$ 并且 $n \geqslant 7$.

如下定义 $n$ 个顶点的 $\frac{n+3-i}{3}-$ 正则 2 - 图:

(a) $n=6 r+4, r \geqslant 1$. 设 $M(6 r+4,2 r+1)$ 是 $n$ 个顶点为

$$
\left\{v_{0}\right\} \cup\left\{v_{i, j} \mid i=1,2, \cdots, d, j=1,2,3\right\},
$$

边为

$$
\begin{gathered}
\left\{v_{0} v_{i, 1} \mid i=1,2, \cdots, d\right\} \cup\left\{v_{i, 1} v_{i, 2}, v_{i, 1} v_{i, 3} \mid i=1,2, \cdots, d\right\}^{(r)} \\
\cup\left\{v_{i, 2} v_{i, 3} \mid i=1,2, \cdots, d\right\}^{(r+1)}
\end{gathered}
$$

的图, 其中 $S^{(r)}$ 记 $r$ 重 $S$ 的多重集 (见图 1(a)), 则 $M(6 r+4,2 r+1)$ 是有 $(2 r+1)$ 条割边的 $(2 r+1)$ - 正则图. 我们有 $2 r+1=(n+3-4) / 3$.

(b) $n=6 r+1, r \geqslant 1$. 如下构造一个 2- 图 $M(6 r+1,2 r+1)$ : 从图 $M(6 r+$ $4,2 r+1$ ) 中删除一个三角形 $\left\{v_{d, 1}, v_{d, 2}, v_{d, 3}\right\}$, 添加一条单边 $\left\{v_{0}\right\}$ (见图 1(b), 其中 环绕顶点的圆圈表示一条单边, 即尺寸为 1 的一条边). 于是 $M(6 r+1,2 r+1)$ 有 $n=6 r+1$ 个顶点, 并且

$$
2 r+1=\frac{(6 r+1)+2}{3}=\frac{n+3-1}{3} .
$$

(c) $n=6 r+2, r \geqslant 1$. 如下构造一个 2- 图 $M(6 r+2,2 r+1):$ 从图 $M(6 r+$ $1,2 r+1)$ 中删除边 $\left\{v_{0}\right\}$, 添加一个新顶点 $v_{6 r+2}$, 一条边 $\left\{v_{0} v_{6 r+2}\right\}$ 和 $2 r$ 重 $\left\{v_{6 r+2}\right\}$ (见图 1(c)). 于是 $M(6 r+2,2 r+1)$ 有 $n=6 r+2$ 个顶点, 并且

$$
2 r+1=\frac{(6 r+2)+3-2}{3}=\frac{n+3-2}{3} .
$$

(d) $n=6 r+5, r \geqslant 1$. 从图 $M(6 r+4,2 r+1)$ 构造一个 2- 图 $M(6 r+5,2 r+1)$, 通过添加一个新的顶点 $v_{6 r+5}$, 添加 $\left\{v_{6 r+5}\right\}$ 的 $2 r+1$ 条单边 (见图 1(d)). 于是 $M(6 r+5,2 r+1)$ 有 $n=6 r+5$ 个顶点, 并且

$$
2 r+1=\frac{(6 r+5)+3-5}{3}=\frac{n+3-5}{3} .
$$

(e) $n=6 r+3, r \geqslant 1$. 构造一个 2- 图 $M(6 r+3,2 r+1)$, 通过从图 $M(6 r+2,2 r+$ 1) 中去掉所有与 $\left\{v_{6 r+2}\right\}$ 关联的边, 添加一个新的顶点 $v_{6 r+3}$, 一条边 $\left\{v_{6 r+3}\right\}$ 和 $2 r$ 重的 $\left\{v_{6 r+2} v_{6 r+3}\right\}$ (见图 1(e)). 于是 $M(6 r+3,2 r+1)$ 有 $n=6 r+3$ 个顶点, 并 且

$$
2 r+1=\frac{(6 r+3)+3-3}{3}=\frac{n+3-3}{3} .
$$

(f) $n=6 r+6, r \geqslant 1$. 从图 $M(6 r+4,2 r+1)$ 构造一个 2- 图 $M(6 r+6,2 r+1)$, 通过添加新顶点 $v_{6 r+5}$ 和 $v_{6 r+r}$, 添加 $2 r+1$ 重的 $\left\{v_{6 r+5} v_{6 r+6}\right\}$ (见图 1(f)). 于是 
$M(6 r+6,2 r+1)$ 有 $n=6 r+6$ 个顶点, 并且

$$
2 r+1=\frac{(6 r+6)+3-6}{3}=\frac{n+3-6}{3} .
$$

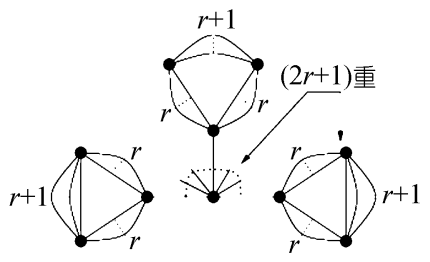

(a) $M(6+4,2 r+1)$

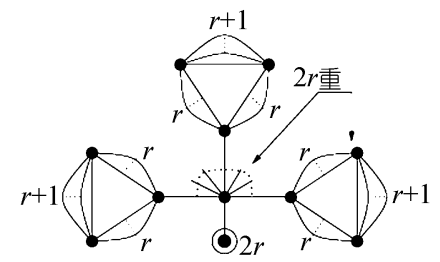

(c) $M(6 r+2,2 r+1)$

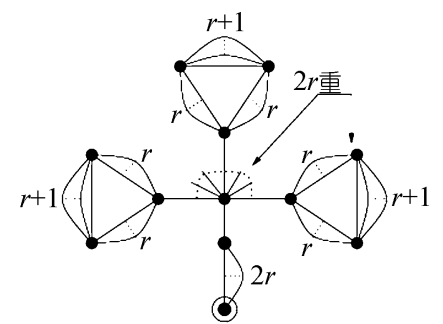

(e) $M(6 r+3,2 r+1)$

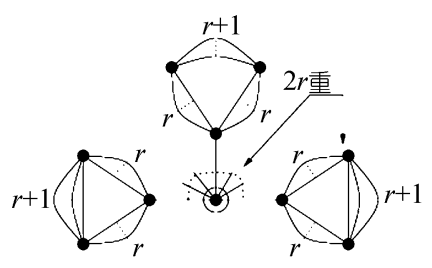

(b) $M(6 r+1,2 r+1)$

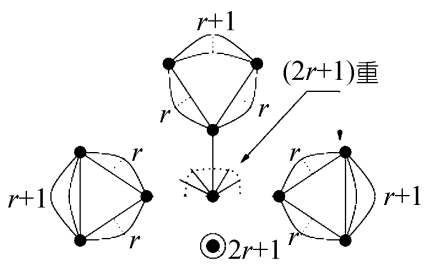

(d) $M(6 r+5,2 r+1)$

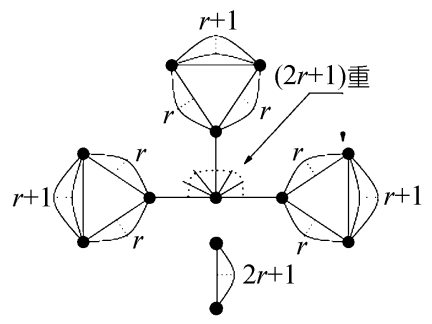

(f) $M(6 r+6,2 r+1)$

图 1 一些极小 $\frac{n+3-i}{3}$ - 正则 2- 图

图 1 给出了以上构造的这些图. 容易看出, 在 (a) (f) 所构造的那些 2- 图中, 由顶点 $v_{0}$ 所导出的那个分支是无 2- 因子的. 同时也有 $(2 r+1)$ 条割边与 $v_{0}$ 邻 接, 因此由定理 2.3, 所有这些 2- 图都是极小的. 这就证明了 $f_{2}(n) \geqslant(n+3-i) / 3$, 其中 $1 \leqslant i \leqslant 6$ 并且 $i \equiv n(\bmod 6)$.

最后证明 $f_{2}(n) \leqslant(n+3-i) / 3,1 \leqslant i \leqslant 6$ 并且 $i \equiv n(\bmod 6)$.

首先, 由以上的构造知道 $M(7,3)$ 是一个极小 3 - 正则 2- 图, 因此 $f_{2}(7) \geqslant 3$. 因为 $n$ 个顶点的极小 $f_{2}(n)$ - 正则 2- 图加上被 $f_{2}(n)$ 条单边所覆盖的另一顶点给 出 $(n+1)$ 个顶点的极小 $f_{2}(n)$ - 正则 2 - 图, 所以当 $n \geqslant 7$ 时, 有

$$
3 \leqslant f_{2}(n) \leqslant f_{2}(n+1) .
$$

此外, 由定理 $2.3, f_{2}(n)$ 是奇的.

设 $G$ 是极小 $f_{2}(n)$ - 正则 2- 图，又可以假设 $G$ 是本原的. 设 $\{x\}$ 是重数为 $p$ 的那条单边. 若 $p=0$, 则 $G$ 是一个图. 假设 $p>0$, 我们需要将 $G$ 转化为一个 $f_{2}(n)$ - 正则图. 
如果 $p=f_{2}(n)$, 那么 $x$ 是一个孤立顶点, 从 $G$ 中删除 $x$. 如果 $p=2 m$, 那么 去掉 $p$ 重 $\{x\}$, 添加新顶点 $y, z$, 添加 $m$ 重 $x y, m$ 重 $x z$ 和 $f_{2}(n)-m$ 重 $y z$; 如果 $p=2 m+1<f_{2}(n)$, 那么去掉 $p$ 重 $\{x\}$, 添加新顶点 $y, z, w$, 添加 $2 m+1$ 重的边 $x y,\left(f_{2}(n)-2 m-1\right) / 2$ 重的 $y z$ 和 $y w$, 以及 $2 m+1$ 重的 $z w$. 设 $G^{\prime}$ 是由以上构造 所得到的图, 那么 $G^{\prime}$ 是一个极小 $f_{2}(n)$ - 正则图, 否则 $G$ 不会是极小的. 注意 $G^{\prime}$ 至多有 $n+3$ 个顶点.

由定理 2.3, $G^{\prime}$ 有一个无 2- 因子的分支 $C$, 它至少与 $f_{2}(n)$ 条割边邻接. 因 为 $G^{\prime}$ 是一个 $f_{2}(n)$ - 正则图并且 $f_{2}(n) \geqslant 3$ 是奇数, 所以每条那样的割边都连接 $G^{\prime}$ 的一个至少 3 个顶点的分支 (不必 2 - 连通). 于是有 $3 f_{2}(n)+|C| \leqslant\left|G^{\prime}\right|$, 因而

$$
f_{2}(n) \leqslant \frac{\left|G^{\prime}\right|-|C|}{3} \leqslant \frac{n+3-1}{3}=\frac{n+2}{3} .
$$

设 $n=6 r+i$, 其中 $r \geqslant 1$ 并且 $1 \leqslant i \leqslant 6$. 由 (4) 式有

$$
f_{2}(n) \leqslant \frac{n+2}{3}=\frac{6 r+i+2}{3}=2 r+1+\frac{i-1}{3} .
$$

因为 $\left\lfloor\frac{i-1}{3}\right\rfloor \leqslant 1$ 并且 $f_{2}(n)$ 是奇的, 从而得到

$$
f_{2}(n) \leqslant 2 r+1=\frac{n+3-i}{3} .
$$

定理证毕.

注 1 (i) 按照已知的 Hilbert 基算法 ${ }^{[5,6]}, f_{2}(n)$ 的值可作为计算 $\mathcal{G}_{2}(n)$ 的一 个终端条件, 因此, 可以得到一个关于 $\mathcal{G}_{2}(n)$ 的更有效的算法.

(ii) 定理 2.3 也蕴含构造 $\mathcal{G}_{2}(n)$ 中所有 2- 图的一个递归算法.

\section{4 关于正则图的结果}

因为图是本原 2- 图, 因此也可如下表述定理 2.3:

定理 4.1 一个 $k$-正则多重图 (无环) $G$ 是不可因子分解的当且仅当下列之 一成立:

(i) $G$ 是一个对集;

(ii) $G$ 是不相交的圈的并, 并且其中一个是奇圈;

(iii) $k(\geqslant 3)$ 是奇的, 并且 $G$ 含有无 2- 因子的分支 $C$ 与 $G$ 的至少 $k$ 条割边 邻接.

上面的特征定理蕴含着以下关于度条件的 2- 因子存在性定理：

定理 4.2 (i) 若 $G$ 是一个 $(2 r+1)$ - 正则多重图, 并有

$$
2 r+1>\frac{|V(G)|-1}{3},
$$

则 $G$ 有 2- 因子.

(ii) 若 $G$ 是一个 $(2 r+1)$ - 正则简单图, 并有 $(2 r+2)^{2}>|V(G)|$, 则 $G$ 有 2因子.

证 假定 $G$ 没有 2- 因子, 则由定理 4.1, G 有一个无 2- 因子分支 $C$, 它至少 与 $(2 r+1)$ 条割边邻接. 每条这样的割边都连接 $G$ 的一个分支 (不必 2 - 连通), 由 
$G$ 的正则性, 当 $G$ 是一个多重图时, 该分支至少有 3 个顶点, 当 $G$ 是简单图时, 该分支至少有 $(2 r+3)$ 个顶点.

因此, 如果 $G$ 是一个多重图, 那么

$$
3(2 r+1)+|C| \leqslant|V(G)|,
$$

所以

$$
2 r+1 \leqslant \frac{|V(G)|-|C|}{3} \leqslant \frac{|V(G)|-1}{3},
$$

这与定理的条件 (i) 矛盾.

若 $G$ 是一个简单图，则

$$
(2 r+3)(2 r+1)+|C| \leqslant|V(G)|,
$$

因此

$$
(2 r+3)(2 r+1)+1 \leqslant|V(G)|,
$$

即

$$
(2 r+2)^{2} \leqslant|V(G)|,
$$

这与定理的条件 (ii) 矛盾.

注 2 图 1(a) 中的图 $M(6 r+4,2 r+1)$ 表明定理 4.2 (i) 是最好的可能. 下 面，设 $H$ 是顶点数为 $2 r+3$ 的简单图，使得一个顶点的度为 $2 r$, 其他顶点的度 为 $2 r+1$ (容易看出这样的图存在). 通过用 $H$ 来替换图 $M(6 r+4,2 r+1)$ 中每一 个重三角形，我们构造出一个图 $G$, 则 $G$ 是简单的、 $(2 r+1)$ - 正则的, 并且有

$$
(2 r+1)(2 r+3)+1=(2 r+1)^{2}
$$

个顶点. 显然 $G$ 没有 2- 因子, 因此定理 4.2 (ii) 也是紧的.

定义 $g_{2}(n)$ 是 $n$ 个顶点的具有最多边数的一个不可因子分解正则图的度数. $g_{2}(n)$ 是确切定义的, 并且 $g_{2}(n) \leqslant f_{2}(n)$. 我们有以下关于 $g_{2}(n)$ 的结果:

\section{定理 4.3}

$$
g_{2}(n)= \begin{cases}0, & n=1, \\ 1, & n=2,4, \\ 2, & n=3,5,6,8, \text { 或 } n \text { 是奇数并且 } n \geqslant 7, \\ 2 r-1, & n=6 r+2, r>1, \\ 2 r+1, & n=6 r+4 \text { 或 } 6 r+6, r>0 .\end{cases}
$$

证 $n \leqslant 8$ 时, 容易验证定理成立. 设 $n$ 是奇数并且 $n \geqslant 7$. 因为一个不可 因子分解的 $g_{2}(n)$ - 正则图 $G$ 的度数和是 $n g_{2}(n)$, 而它是偶数, 所以 $g_{2}(n)$ 必定为 2. 否则, 由引理 2.3, $G$ 是 2- 因子可分解的.

设 $n=6 r+2, r>1$. 我们有

$$
g_{2}(n) \leqslant f_{2}(n)=2 r+1 .
$$


若 $g_{2}(n)=2 r+1$, 则有一个无 2- 因子的分支 $C$ 与至少 $(2 r+1)$ 条割边邻接. 每 条割边连接 $G$ 的一个至少有 3 个顶点的分支 (除了 $C$ ). 这意味着至少有

$$
3(2 r+1)+|C| \geqslant 6 r+4
$$

个顶点, 矛盾. 另外, 注意到由引理 2.3 , 当 $r>1$ 时, $g_{2}(6 r+2) \neq 2 r$, 因此 $g_{2}(6 r+2) \leqslant 2 r-1$. 下面, 我们构造一个 $(6 r+2)$ 个顶点的不可因子分解 $(2 r-1)-$ 正则图, 从而证明 $g_{2}(6 r+2)=2 r-1$.

一个几乎 $k$-正则 $m$ - 圈是有重边的 $m$ - 圈, 使得圈中除 1 个顶点以外, 其他 所有顶点的度都为 $k$, 排除在外的那个顶点的度为 $k-1$, 称其为根. 容易看出存 在几乎 $(2 r-1)$ - 正则 3- 圈和 5- 圈. 设 $v_{0}$ 是一个顶点, 它与两个几乎 $(2 r-1)$ - 正 则 5- 圈和 $(2 r-3)$ 个几乎 $(2 r-1)$ - 正则 3- 圈在根上连接, 所得的图是 $(6 r+2)$ 个顶点的 $(2 r-1)$ - 正则图, 它是不可因子分解的.

因为

$$
g_{2}(6 r+4) \leqslant f_{2}(6 r+4)=2 r+1, \quad g_{2}(6 r+6) \leqslant f_{2}(6 r+6)=2 r+1,
$$

并且 $M(6 r+4,2 r+1)$ (图 1(a)) 和 $M(6 r+6,2 r+1)$ (图 1(f)) 是不可因子分解的 图, 从而得到

$$
g_{2}(6 r+4)=g_{2}(6 r+6)=2 r+1
$$

\section{$5 f_{s}(n)$ 及尚未解决的问题}

作为推广, 考虑边尺寸至多为 $s(s \geqslant 2)$ 的超图, 并称它们为 $s$ - 图. 设 $2 \leqslant$ $s \leqslant n$, 我们用 $\mathcal{G}_{s}(n)$ 记顶点集 $\{1,2, \cdots, n\}$ 上所有极小正则 $s$ - 图的集合, 并用 $f_{s}(n)$ 记 $\mathcal{G}_{s}(n)$ 中所有 $s$ - 图度数的最大值, 特别地, 令

$$
f(n)=f_{n}(n), \quad \mathcal{G}(n)=\mathcal{G}_{n}(n),
$$

则 $\mathcal{G}_{s}(n)$ 和 $f_{s}(n)$ 都是有限的. 显然

$$
\begin{gathered}
\mathcal{G}_{2}(n) \subset \mathcal{G}_{3}(n) \subset \cdots \subset \mathcal{G}_{n}(n)=\mathcal{G}(n), \\
f_{2}(n) \leqslant f_{3}(n) \leqslant \cdots \leqslant f_{n}(n)=f(n) .
\end{gathered}
$$

$\mathcal{G}(n)$ 和 $f(n)$ 可应用于超通用开关盒的设计 ${ }^{[9]}$. 已经知道 ${ }^{[9]}$

$$
f(1)=1, \quad f(2)=1, \quad f(3)=2, \quad f(4)=3, \quad f(5)=5, \quad f(6)=9 .
$$

在一个 LDES $A X=0$ 的极小解 $\left(x_{1}, \cdots, x_{q}\right)$ 的分支和上运用 Pottier 的上界 [10]

$$
\sum_{i=1}^{q} x_{i, j} \leqslant \prod_{i=1}^{p}\left(\sum_{j=1}^{q} a_{i, j}+1\right),
$$

容易得到

$$
f(n) \leqslant 2\left(2^{n-1}+1\right)^{n} / n .
$$


然而, 这个上界对于实际应用来说太大了. 函数 $f_{s}(n)$ 和 $\mathcal{G}_{s}(n)$ 中图的研究对于 具有特殊性质的开关盒的设计, 既有理论意义, 也有实际应用. 我们提出下面的 问题:

问题 对 $s \geqslant 3$ 和 $n \geqslant 7$, 确定 $f_{s}(n)$ 的值.

\section{6 在一般 USB 设计中的应用}

本节简要描述 $f_{2}(n)$ 和极小 2- 图在一般通用开关盒的设计中的应用, 其他 有关的结果可在文献 $[9,11,12]$ 中找到.

可编程门阵列电路 (FPGA) 是一种超大规模集成 (VLSI) 电路, 由线路开关 组成的开关盒是其关键的部分. 在此领域, 一个典型的问题是最大化线路的效 率 (通过改编开关模型中开关的方式制作一个 FPGA 芯片以表现某种功能性的概 率) 和最小化开关模型中开关的数目. 有 $n$ 个面并且每个面上有 $w$ 个终端的开关 记作 $(n, w)$-SB. 一个 $(n, w)-\mathrm{SB}$ 的 $n$ 个面分别标号为 $1,2, \cdots, n$. 用 $v_{j}^{i}$ 记第 $i$ 个面 上的第 $j$ 个终端, 用 $v_{j}^{i} v_{k}^{l}$ 记连接终端 $v_{j}^{i}$ 和 $v_{k}^{l}$ 的开关, 那么一个 $(n, w)-\mathrm{SB}$ 就是 一个 $n$ 部图 $\left(V^{1}, \cdots, V^{n}, E\right)$, 其中 $V^{i}=\left\{v_{j}^{i} \mid 1 \leqslant j \leqslant n\right\}, E$ 是开关的集合.

一个常规需求 (限制在一个 $n$ 面开关盒上) 是一个线路布局, 它由网络构成, 每个网络象征开关盒的两个面, 在线路中这两个面将由一个开关连接. 我们现在 可以把一个常规需求抽象成 $\{1,2, \cdots, n\}$ 的基数为 2 的一个子集族. 为了简单化和 规律性, 加入一些单边以确保 $\{1,2, \cdots, n\}$ 中的每个元出现的次数相同, 因此, 一 个常规需求就变成了一个正则 2- 图. 在一个 $(n, w)-\mathrm{SB} B\left(n\right.$ 部图 $\left.\left(V^{1}, \cdots, V^{n}, E\right)\right)$ 中, 如果存在 $B$ 的一个对集 $M$, 以及 $G$ 的非单边边集 $E_{2}$ 与 $M$ 之间的一个一一 对应, 使得任意的 $i j \in E_{2}$ 与 $M$ 中连接 $V^{i}$ 中一个顶点和 $V^{j}$ 中一个顶点的一条 边相对应，那么在顶点集 $\{1,2, \cdots, n\}$ 上的一个 (常规需求) 正则 2- 图 $G$ 就称为 是规范的, $M$ 称为 $B$ 中 $G$ 的一个部分线路. 通用开关盒的设计问题可表述为 设计一个最小规模的每一部含 $w$ 个顶点的 $n$ 部图, 它含有一个部分程序对应于 任意有 $n$ 个顶点的 $w$-正则 2- 图. 对任意有 $n$ 个顶点的 $w$-正则 2- 图是规范的 $(n, w)-\mathrm{SB}$ 记作 $(n, w)$-USB. 一个显而易见的解答是各部分具有 $w$ 个顶点的完全 $n$ 部图, 然而它含有 $\left(\begin{array}{l}n \\ 2\end{array}\right) w^{2}$ 个开关.

文献 [13] 中给出了最优的 $(4, w)$-USB 设计. 文献 [12] 中宣称设计出了最优 的 $(n, w)-\mathrm{USB}$, 不幸的是, 如文献 [11] 所指出的那样, 它是不正确的. 一般的最 优 USB 设计问题尚未解决.

运用本文的结果, 可以得到 $(n, w)-\mathrm{USB}$ 的一个好的近似设计. 由定理 3.1 和 2.2 , 我们有下述程序要求分解定理和 USB 合成定理:

定理 6.1 设 $G$ 是有 $n$ 个顶点的 $w$-正则 2- 图, 则有

(i) 当 $w$ 是偶数时, $G$ 可分解成 $\frac{w}{2}$ 个 2- 因子.

(ii) 当 $1 \leqslant n \leqslant 6$ 并且 $w$ 是奇数时, $G$ 可分解成一个 1 - 因子和 $\frac{w-1}{2}$ 个 2 - 因 
子.

(iii) 当 $n \geqslant 7, w$ 是奇数并且 $w>\frac{k+3-i}{3}$ 时, $G$ 可分解成一个 $\frac{n+3-i}{3}$ - 因子 和 $\frac{3 w-n-3+i}{6}$ 个 2 - 因子, 其中 $1 \leqslant i \leqslant 6$ 并且 $i \equiv n(\bmod 6)$. 当 $n \geqslant 7$ 并且奇数 $w \leqslant \frac{n+3-i}{3}$ 时, 对每一对 $n, w$ 均存在一个极小 $w$ - 正则 2- 图.

根据这个程序要求分解定理, 当 $w\left(\geqslant \frac{n+3-i}{3}\right)$ 是奇数并且 $n \geqslant 7$ 时, 要设计一 个 $(n, w)-\mathrm{USB}$, 只需设计一个 $(n, 1)-\mathrm{USB}$, 一个 $(n, 2)-\mathrm{USB}$ 和一个 $\left(n, \frac{n+3-i}{3}\right)-\mathrm{USB}$. 因此，关于 $(n, w)-\mathrm{USB}$ 的设计我们有以下对应的定理:

定理 6.2 (i) 若 $w$ 是偶数, $\frac{w}{2}$ 个 $(n, 2)-\mathrm{USB}$ 的不相交的并形成一个 $(n, w)-$ USB.

(ii) 若 $1 \leqslant n \leqslant 6$ 并且 $w$ 是奇数, 一个 $(n, 1)-\mathrm{USB}$ 和 $\frac{w-1}{2}$ 个 $(n, 2)-\mathrm{USB}$ 的不 相交的并形成一个 $(n, w)-\mathrm{USB}$.

(iii) 若 $n \geqslant 7$, 并且奇数 $w>\frac{n+3-i}{3}$, 则一个 $\left(n, \frac{n+3-i}{3}\right)-\mathrm{USB}$ 和 $\frac{3 w-n-3+i}{6}$ 个 $(n, 2)-\mathrm{USB}$ 的不相交的并形成一个 $(n, w)-\mathrm{USB}$. 当 $n \geqslant 7$, 并且奇数 $w \leqslant \frac{n+3-i}{3}$ 时, 一个 $(n, w)$-USB 必定是连通的.

文献 [3] 中设计出了最优的 $(n, 1)-\mathrm{USB}$ 和 $(n, 2)-\mathrm{USB}$, 它们分别有 $\frac{n(n-1)}{2}$ 和 $n(n-1)$ 个开关. 当 $n \geqslant 7$ 并且 $w$ 是奇数时, 文献 [3] 也给出了一个方法来构造 几乎最优的 $(n, w)$-USB. 然而, 即使对一个完全 $\left(n, \frac{n+3-i}{3}\right)$-USB 而言, 用定理 6.2 (iii) 构造的一个 $(n, w)-\mathrm{USB}$ 中开关的总数也有

$$
\begin{aligned}
& \frac{3 w-n-3+i}{6} n(n-1)+\frac{n(n-1)}{2}\left(\frac{n+3-i}{3}\right)^{2} \\
= & \frac{n(n-1)}{2} w+\frac{n(n-1)}{2} \frac{n+3-i}{3} \frac{n-i}{3} \\
= & O(w),
\end{aligned}
$$

反之，一个完全 $(n, w)$-USB 有

$$
\frac{n(n-1)}{2} w^{2}=O\left(w^{2}\right)
$$

个开关.

注意到已经得知 $(n, w)-\mathrm{USB}$ 的开关数目的一个下界是 $\frac{n(n-1)}{2} w^{[3]}$, 因此, 当 $w$ 很大时, 对一个固定的 $n$, 由定理 6.2 (iii) 得到的 $(n, w)-\mathrm{USB}$ 中的开关的数目与 一个最优 $(n, w)$-USB 的开关的数目之比接近于 1 .

\section{参考文 献}

1 Bondy J A, Murty U R S. Graph Theory with Applications. London: Macmillan Press, 1976

2 Berge C. Graphs and Hypergraphs. New York: Elsevier Science Publication Company, Inc, 1985

3 Fan H, Liu J, Wu Y L, et al. Reduction design for generic universal switch blocks. ACM Transactions on Design Automation of Electronic Systems (TODAES), 2002, 7(4): 526 546

4 Higman G. Ordering by divisibility in abstract algebras. Proc London Math Soc, 1952, 2 (3): 326 336

5 Contejean E, Devie H. An efficient incremental algorithm for solving systems of linear Diophantine equations. Inform and Comput, 1994, 113(1): 143 172 
6 Tutte W T. The factorization of linear graphs. J London Math Soc, 1947, 22(1): 107 111

7 Petersen J. Die Theorie der regulären graphs. Acta Math, 1891, 15(2): 193 220

8 Akiyama J, Kano M. Factors and factorization of graphs - A survey. J Graph Theory, 1985, 9(1): $67 \sim 86$

9 Fan H, Liu J, Wu Y L. General models and a reduction design technique for FPGA switch box designs. IEEE Transactions on Computers, 2003, 52(1): 21 30

10 Pottier L. Minimal solutions of linear Diophantine systems: bounds and algorithms. In: Book R V, ed. Proceedings of the 4th International Conference on Rewriting Techniques and Applications, Lecture Notes in Computer Science, Vol 488. Berlin: Springer, 1991. 162 173

11 Fan H, Wu Y L, Chang Y W. A comment on "General universal switch blocks". IEEE Transactions on Computers, 2002, 51(1): 93 95

12 Shyu M, Wu G M, Chang Y D, et al. Generic universal switch blocks. IEEE Trans on Computers, 2002, 51(3): 348 359

13 Chang Y W, Wong D F, Wong C K. Universal switch models for FPGA. ACM Trans on Design Automation of Electronic Systems, 1996, 1(1): 80 101 